\title{
Identidades enredadas en el tejido del tiempo: La metáfora futurista de Wong Kar-Wai
}

\author{
Identities tangled in the cloth of time: \\ Wong Kar-Wai's futuristic metaphor
}

\author{
ALFONSO MUÑOZ CORCUERA \\ Departamento de Teoría del Conocimiento, Estética e Historia del Pensamiento \\ Universidad Complutense de Madrid (España)
}

\section{RESUMEN}

El cine de Wong Kar-Wai es, principalmente, un cine sobre el tiempo. El director chino explora en sus películas la relación del sujeto con la temporalidad en la que se encuentra, mostrando el complejo entramado que une el pasado, el presente y el futuro en una tela que, al igual que la de Penélope, se teje tanto como se desteje. Quizá el mejor exponente de esta situación sea esa suerte de trilogía formada por Días salvajes (1990), Deseando amar (2000) y 2046 (2004), unida no por su argumento sino por la temática que abordan: el conflicto que viven unos personajes al encontrarse atrapados dentro de una temporalidad que sienten ajena o injusta.

\section{PALABRAS CLAVE}

WONG KAR-WAI, 2046, MEMORIA, IDENTIDAD

\begin{abstract}
The films by Wong Kar-Wai are primarily about time. This Chinese director explores in his films the subject's relationship to the temporality in which he is, showing the complex network that links the past, the present and the future in a cloth which, like that of Penelope's, is as much
\end{abstract}


woven as unravelled. Perhaps the best example of this situation is that sort of trilogy formed by Days of Being Wild (1990), In the Mood for Love (2000) and 2046 (2004), linked not by their argument but because they address the same issue: the conflict that the characters live since they are trapped within a temporality that they feel alien or unfair.

KEYWORDS

WONG KAR-WAI, 2046, MEMORY, IDENTITY

Es asombroso: ahí está el instante presente, pero en un abrir y cerrar de ojos desaparece. Surge de la nada para desaparecer en la misma nada. Sin embargo, luego regresa como un fantasma perturbando la calma de un presente posterior. ${ }^{1}$

\section{INTRODUCCIÓN}

LA TRILOGÍA CINEMATOGRÁFICA QUE El DIRECTOR CHINO Wong Kar-Wai comenzó con Días salvajes, y que continuó con la exitosa Deseando amar, llegó a un sorprendente cierre con la película 2046. En este último film se mezclan las dolorosas historias del pasado, procedentes de las dos primeras entregas de la trilogía, con el extraviado presente de su protagonista y con las confusas expectativas de futuro del mismo, presentadas en clave de relato de ciencia-ficción. En este sentido, la memoria se constituye como uno de los elementos centrales en el desarrollo de 2046, pues el pasado adquiere tal importancia en el presente del protagonista que le lleva incluso a imaginar arruinado su futuro. De este modo se muestra cómo el sentimiento de pérdida que el protagonista experimentaba al final de Deseando amar, aunque resultó en un principio aliviado gracias a la búsqueda del olvido, sigue actuando de forma inconsciente en su presente. El trabajo de duelo que debía guiarle en su camino se pervirtió antes de finalizar, dando lugar a un estado doloroso e inesperado.

En las próximas páginas trataré de mostrar a partir del análisis de 2046 cómo se desarrolla este conflicto, qué papel juega la memoria en la vida del personaje protagonista y cómo afecta a su identidad.

1 F. Nietzsche 2003, p. 41. 


\section{LA MEMORIA Y LA POLARIDAD PASADO-FUTURO}

En La lectura del tiempo pasado, Paul Ricoeur trata de analizar las características que, a su juicio, posee la memoria, señalando cuatro aspectos que considera fundamentales: el carácter propio, la continuidad, la polaridad pasado-futuro y la pretensión veritativa. Estas cuatro características serían las garantes que permitirían asegurar la residencia de una identidad fija en la memoria. El carácter propio, explica Ricoeur, se refiere al hecho de que «no pueden transferirse los recuerdos de uno a la memoria de otro». ${ }^{2}$ De este modo se asegura que la identidad de un individuo no puede confundirse con la de nadie más. ${ }^{3}$ La continuidad hace referencia al vínculo que se establece entre el presente y el pasado gracias a la memoria, que nos permite remontarnos sin solución de continuidad desde el presente vivido hasta los acontecimientos más lejanos de nuestra infancia. De ese modo, la memoria garantiza la continuidad temporal de la persona a la vez que permite ubicar en el tiempo los distintos recuerdos. ${ }^{4}$ Por su parte, la polaridad pasado-futuro, muy relacionada con la idea de continuidad, es la sensación de orientarse a lo largo del tiempo, del pasado al futuro. Esta característica se da gracias a la interacción de los conceptos de «espacio de experiencia» y de «horizonte de espera» definidos por Reinhart Koselleck en Futuro pasado: para una semántica de los tiempos históricos. El espacio de experiencia, como conjunto de herencias del pasado, configura un cierto horizonte de espera con respecto al futuro. Además, gracias a este horizonte de espera, la experiencia del presente adquiere un grado de sentido o

2 P. Ricoeur 1999, p. 16.

3 Por poner un ejemplo conocido de crítica a este tipo de afirmaciones puede pensarse en la película Blade Runner (R. Scott, dir., 1982), en la que una empresa se dedica a fabricar una especie de clones de seres humanos que poseen los recuerdos de la persona original. Una crítica filosófica más exhaustiva es la que llevó a Shoemaker a la elaboración del concepto de cuasimemoria, término aplicable a los recuerdos en los que el sujeto recordador y el sujeto recordado no coinciden. $C f$. S. Shoemaker 1970.

4 La continuidad de la memoria fue precisamente el criterio de identidad personal que defendió Locke en su Ensayo sobre el entendimiento humano. Sin embargo ya en el siglo XVIII se encuentra una crítica de peso a esta idea. Thomas Reid argumentó que la memoria no puede ser la base de la identidad, pues a diferencia de ésta, la memoria no es una propiedad transitiva, para lo cual tendría que tener una continuidad de la que carece. Podemos imaginar un sujeto que recuerda perfectamente un suceso de su vida acaecido hace 10 años, pero que sin embargo ha olvidado otro suceso que sucedió hace 20 , a pesar de que hace 10 años sí lo recordaba. En este caso, si la memoria fuese realmente un criterio para determinar la identidad de una persona, tendríamos que aceptar que el sujeto en cuestión al mismo tiempo es y no es la misma persona que era hace 20 años. Como esto es un absurdo, la memoria no puede ser el criterio de la identidad personal porque carece de una verdadera continuidad. Para una discusión posterior de este argumento $c f$. D. Parfit 2004, pp. 381-383. 
de sinsentido que a su vez «otorga a la conciencia histórica su valor cualitativo, irreductible a la dimensión meramente cronológica del tiempo». ${ }^{5}$ Por lo tanto, gracias a la polaridad pasado-futuro el hombre sería capaz de aprovechar sus conocimientos del pasado para prever y planificar su futuro, dotando así de un sentido a su experiencia presente. Finalmente, Ricoeur introduce la noción de pretensión veritativa como característica de la memoria para diferenciarla de la imaginación: para el filósofo francés, la memoria desea y asume la labor de ser fiel y exacta, lo que no es incompatible con el hecho de que en ocasiones los recuerdos puedan no reflejar fielmente los sucesos del pasado. Las discrepancias que pudiesen encontrarse se deberían entender como simples errores de la memoria en su tarea de preservar intacto el pasado.

Esta concepción de la memoria expresada por Ricoeur es discutible en muchos aspectos. El principal problema radica en que Ricoeur insiste en defender la existencia de una identidad única y estable para cada individuo, lo que, desde la psicología posmoderna, es imposible defender. ${ }^{6}$ Esto le lleva a atribuir a la memoria unas características que chocan frontalmente con la realidad, como en el caso más evidente de la pretensión veritativa. ${ }^{7}$ Sin embargo, a pesar de que podría analizarse cómo se ven cuestionadas todas estas características de la memoria en 2046, me gustaría centrarme en la polaridad pasado-futuro, por ser la más interesante desde mi punto de vista para analizar la película de Wong Kar-Wai.

Si entendemos esta característica simplemente como la sensación de orientarse a lo largo del tiempo, del pasado al futuro, poco se podría objetar, pues evidentemente existe una sensación en el hombre de estar orientado de este modo. Sin embargo, sí me gustaría hacer algunos comentarios respecto a la consecuencia que se extraía de la reflexión de Ricoeur -la de que el hombre es capaz de aprovechar sus conocimientos del pasado para prever y planificar su futuro, dando así un sentido a su experiencia presente-, ya que debe ser interpretada dentro de un marco restringido. Pero antes de seguir adelante, considero importante destacar la intención que tenía Ricoeur al atribuir esta característica a la memoria, ya que su complejidad hace que necesite una aclaración más extensa para ser comprendida en su totalidad.

5 Ibid., p. 22.

6 En este sentido el psicólogo Kenneth J. Gergen asegura que en la postmodernidad «el yo se desvanece totalmente y desaparece en un estado de relacionalidad. Uno cesa de creer en un yo independiente de las relaciones en que se encuentra inmerso» (K. J. Gergen 1992, p. 38).

7 El hecho de que la memoria es, fundamentalmente, subjetiva y por tanto no es ni pretende ser un registro fiel, sino una interpretación del pasado, es algo aceptado no sólo por corrientes psicológicas como el constructivismo o el psicoanálisis, sino incluso desde la neurociencia. $C f$. E. R. Kandel 2007 y D. C. Dennett 1995. 
El problema para Ricoeur, a la hora de plantear su estudio, era obsesionarse «con el "carácter pasado" del propio pasado», ${ }^{8}$ por lo que decidió hacer hincapié en la importancia del futuro en la concepción de éste. El horizonte de espera -como expectativa de futuro- dota de un sentido o sinsentido a las acciones presentes en la medida en que éstas se dirigen a la consecución de algún fin concreto situado en ese horizonte, al mismo tiempo que modifica nuestra idea del pasado, ya que éste es reinterpretado en función de los objetivos futuros que se quieren alcanzar. En este sentido, la polaridad pasado-futuro de la memoria hace referencia no sólo a la sensación de orientarse a lo largo del tiempo, sino también a la interacción que se produce entre la memoria, que nos hace crearnos unas expectativas con respecto al futuro, y las propias expectativas de futuro, que modifican a su vez el sentido de la experiencia presente y pasada.

Las complicaciones aparecen a la hora de interpretar la naturaleza de estas influencias mutuas entre la memoria, la experiencia presente y las expectativas de futuro. Han de tenerse en cuenta tres cuestiones que Ricoeur no considera a la hora de interpretar dichas influencias:

1. Los recuerdos que constituyen el espacio de experiencia, además de no ser fiables, pueden interferir en la experiencia presente y en la creación del horizonte de espera no sólo en un sentido positivo, sino también negativo, y de un modo tanto consciente como inconsciente.

2. El sentido o sinsentido que el horizonte de espera confiere a la experiencia presente no puede interpretarse como algo absoluto, sino relativo, ya que sólo es válido para el propio individuo.

3. La influencia del horizonte de espera en el espacio de experiencia puede constituir un atentado a la pretensión veritativa de la memoria.

Dejando de lado las cuestiones relativas a la pretensión veritativa de la memoria y al sentido que el horizonte de espera confiere a la experiencia presente, podemos analizar 2046 atendiendo al modo en que el espacio de experiencia puede influir en las acciones del presente y en la creación del horizonte de espera.

La película de Wong Kar-Wai, cuya trama es una continuación de Deseando amar focalizada en uno de los personajes principales, el escritor y periodista Chow Mo-Wan, nos presenta dos historias paralelas: por un lado, la película nos cuenta la vida de Chow después de la fracasada experiencia amorosa de

8 P. Ricoeur op. cit., p. 23. 
Deseando amar; por otro, una historia de ciencia-ficción titulada «2046» que Chow ha escrito para exorcizar los fantasmas de su pasado. En un cambio que al principio parece incomprensible para el espectador, el tierno Chow de Deseando amar se ha convertido en 2046 en un cínico mujeriego. Estas personalidades son prácticamente antagónicas, y mientras en la primera película se nos presentaba el cálido amor que unía a los dos personajes principales, en la segunda se nos cuentan las múltiples aventuras amorosas de Chow en su nueva vida con mujeres a las que abandona rápidamente sin ningún tipo de remordimiento. Es para comprender este cambio de actitud y personalidad para lo que se hace necesaria la aportación que nos ofrece el relato de ciencia-ficción escrito por Chow, ya que tal y como reconoce explícitamente él mismo, el protagonista del relato es su alter-ego: «Así que empecé a imaginarme que yo era un japonés en un tren rumbo al año 2046 que se enamoraba de una androide reaccionando con efecto retardado».9

Pero ¿qué significa ese viaje en tren para Chow? La primera clave para comprender el simbolismo del viaje al año 2046 se encuentra ya en la escena que da comienzo a la película, en la que la voz en off del protagonista nos lee el primer párrafo de su relato:

En el año 2046, una amplia red de ferrocarriles se extiende por todo el planeta Tierra. De vez en cuando, un tren misterioso parte rumbo a 2046. Todos los pasajeros que se dirigen a ese lugar tienen el mismo objetivo: quieren recuperar la memoria perdida, pues en 2046 nunca cambia nada. Nadie sabe realmente si eso es cierto, porque nadie, absolutamente nadie ha regresado nunca... Excepto yo. ${ }^{10}$

Del monólogo se desprende que el año 2046 es una metáfora del mundo de los recuerdos, un lugar en el que no se sabe si todo es realmente igual a como ocurrió: de nuevo se tambalea la pretensión veritativa de la memoria defendida por Ricoeur. La segunda clave para comprender el simbolismo del viaje a 2046, la que nos da pie para interpretar el resto de la película, se encuentra en la escena final, en la que se repite casi el mismo monólogo que acabo de transcribir, pero con dos pequeñas variaciones: por un lado, las imágenes que acompañan al monólogo ya no son futuristas como al principio, sino que nos muestran al propio Chow, lo que indica que las palabras se refieren a él mismo y no a su personaje; por otro lado, desaparece el «excepto yo» final, lo que nos lleva a pensar que Chow, a diferencia de su personaje, no ha sido capaz de regresar del mundo de los recuerdos, de 2046.

Puede parecer contradictorio que el mundo de los recuerdos se sitúe en un futuro lejano, pero es algo que está plenamente justificado. El año elegido por

9 W. Kar-Wai (dir.) 2004.

10 Ibid.

Contrastes vol. XVIII (2013) 
Chow para situar su relato no es casual, ya que 2046 es el número de la habitación de hotel en la que quedaba con su pretendida en Deseando amar. Es también el número de la habitación que quiere reservar en la nueva película, pero que se encuentra ocupado por otra de las mujeres con las que mantendrá una fugaz relación. Es el símbolo del pasado que se repite en el futuro. Chow está marcado por un pasado que no ha sido capaz de asimilar y que, de modo inconsciente, le lleva a actuar repitiendo compulsivamente la ruptura de su amor en Deseando amar con cada nueva mujer con la que se relaciona. Nuestro protagonista no piensa explícitamente en su antiguo amor. Siguiendo a Freud «podemos decir que [...] no recuerda nada de lo olvidado o reprimido, sino que lo vive de nuevo. No lo reproduce como recuerdo, sino como acto; lo repite sin saber, naturalmente, que lo repite». ${ }^{11}$ El horizonte de espera de Chow está marcado por su espacio de experiencia de un modo inconsciente, pero esto no le produce ningún beneficio, como se podría esperar a partir de los enunciados de Ricoeur, sino al contrario: el hecho de que su pasado condicione su presente y su futuro es una carga de la que desearía liberarse, desearía poder escapar de 2046. El comportamiento que tuvo en el pasado para olvidar a la mujer de Deseando amar, si bien parecía ser útil en un primer momento, no le sirve como experiencia para planificar su futuro porque se revela fallido. Chow trata de llevar a cabo un trabajo de duelo, pero queda sumido en la melancolía, ${ }^{12}$ incapaz de superar la experiencia de pérdida que ha sufrido. Sencillamente no puede olvidar.

\section{LA NOSTALGIA Y LOS RECUERDOS OBSESIVOS}

Hasta ahora hemos visto cómo la pérdida que Chow sufrió en Deseando amar le marcó de una forma tan profunda que le condenó a repetir en el presente la misma experiencia de pérdida de un modo inconsciente y compulsivo. Al final de Deseando amar, Chow trataba de comenzar un trabajo de duelo para olvidar a su gran amor. Sin embargo el protagonista de la película fracasa en su intento, mostrándose incapaz de asimilar un pasado que de este modo pasa a ocupar un lugar central en su experiencia presente. La memoria adquiría tal importancia en la vida de Chow, que su presente y su futuro quedaban irremediablemente condenados a ser una mera repetición del pasado. Envuelto en este conflicto, la única salida para Chow sería, tal y como señala Braude, permitir que el pasado se vuelva memoria (es decir, que deje de vivirlo como un presente activo). ${ }^{13}$ Sólo entonces quedaría liberado del pasado. Seguir adelante no es olvidar o matar el pasado, como intentaba hacer al final de Deseando amar, sino comprender que recordar remite al pasado con uno mismo como «recordador» ubicado en

11 S. Freud 2006a, p. 1684.

12 Sobre la relación entre el duelo y la melancolía, véase S. Freud 2006 b.

13 D. Braude 2007. 
el presente. Sería precisamente el momento en el que el fracaso por resucitar el pasado es total, cuando el tiempo aparecería como vivencia y Chow podría separarse de sus dobles y sus fantasmas. Sin embargo, Chow no transforma el pasado en memoria, lo que hace necesario analizar la situación no desde las características de la memoria, sino desde los usos que se hacen de ella.

Si decimos que Chow hace uso del pasado para su vida, no estaríamos revelando ningún misterio, ya que se desprende como algo evidente de todo lo dicho hasta ahora. Sin embargo el pasado puede ser utilizado de modos muy variados, por lo que la afirmación no nos llevaría a ninguna parte si no matizamos de qué modo Chow hace uso de él. En Sobre los usos y perjuicios de la historia para la vida, Nietzsche señala tres modos de utilizar la historia: el modo anticuario, el modo monumental y el modo crítico. Basándome en esta clasificación, propongo tres modos de utilizar la memoria individual: el nostálgico (basado en el anticuario), el analógico (basado en el monumental) y el negador (basado en el crítico). Me centraré en el modo nostálgico, que es el que caracteriza a Chow.

Para el filósofo alemán, el modo anticuario de abordar la historia otorga a todo lo pasado «su propia dignidad e inviolabilidad», ${ }^{14}$ a la vez que subestima «todo lo que no reconoce el carácter venerable de lo viejo, es decir, todo lo que es nuevo y está en continuo cambio». ${ }^{15}$ Este modo de utilizar la historia coincide en gran medida con lo que Todorov denomina una lectura literal del acontecimiento recuperado. Este tipo de lectura preserva el pasado «en su literalidad (lo que no significa su verdad), permaneciendo intransitivo y no conduciendo más allá de sí mismo». ${ }^{16}$ Por su parte Walter Benjamin denomina historicista a la aproximación al pasado que considera necesario que el historiador «se quite de la cabeza todo lo que sepa sobre lo que ocurrió después» ${ }^{17}$ del acontecimiento que está recuperando. Señala Benjamin que «el historicismo postula una imagen "eterna" del pasado», ${ }^{18}$ lo que significa que lo considera como algo acabado y siempre igual a sí mismo, aunque su interpretación pueda cambiar en función del tiempo y del observador. Recogiendo todas estas características, el modo en que Chow utiliza pasado, que llamo nostálgico, agruparía los usos que descuidan o relegan el momento presente a favor del tiempo recordado, que adquiere el protagonismo.

Podemos ver que el problema que produce el historicismo es exactamente el mismo que le ocasiona a Chow el abuso nostálgico de la memoria: la perpetua-

14 F. Nietzsche, op. cit., p. 60.

15 Ibid., p. 63.

16 T. Todorov 2000, p. 30.

17 W. Benjamin 2006, p. 129.

18 Ibid., p. 249. 
ción del pasado en el presente. Y en este sentido, el comentario que hace Reyes Mate a Benjamin es directamente aplicable a la historia de Chow Mo Wan:

Lo catastrófico en nuestro caso es que «esto» no tenga final, que no haya quien lo pare, que la historia siga con la misma lógica. Lo catastrófico es la eternización de lo que ya tenemos, la irreversibilidad del curso que nos ha traído hasta aquí. Lo angustioso no es que la historia tenga un fin, sino que no lo tenga. ${ }^{19}$

Lo angustioso -podríamos añadir- es ir a 2046 y no poder regresar.

Si bien es Benjamin el autor que más hincapié hace en el problema que supone la perpetuación del pasado, no es el único. Todorov también señala que al hacer una lectura literal del pasado, éste es incapaz de producir nada más allá de sí mismo, ya que extiende «las consecuencias del trauma inicial a todos los instantes de la existencia». ${ }^{20} \mathrm{Y}$ en este mismo sentido creo que se pueden interpretar algunas de las advertencias que Nietzsche hacía de la sobresaturación histórica ${ }^{21}$ que según él invadía su época, demasiado volcada en el modo anticuario:

La sobresaturación histórica de una época me parece que es peligrosa y enemiga de la vida en cinco aspectos: en primer lugar, tal exceso produce ese contraste del que ya hemos hablado entre lo interior y lo exterior por medio del cual se debilita la personalidad; en segundo lugar, da origen a la creencia de poseer la virtud -la más rara de todas- del sentido de la justicia, en un grado superior al de otras épocas; por otro lado, igualmente, se perturban los instintos de un pueblo y se impide llegar a la madurez al individuo, no menos que al conjunto de la sociedad; también crece esa perjudicial creencia de cualquier época de estar en la vejez de la humanidad, de ser mero descendiente y epígono; y, finalmente, cae la época en una peligrosa actitud irónica sobre sí misma, pasando de ésta a una aún más peligrosa: el cinismo. Actitud ésta que evoluciona hacia una acción egoísta que, paralizando al principio, termina destruyendo las fuerzas vitales. ${ }^{22}$

Es decir, el exceso de conocimiento histórico provoca que los individuos no sean capaces de alcanzar una capacidad de decisión propia de un adulto,

19 R. Mate, 2006, p. 163.

20 T. Todorov, op. cit., p. 31.

21 Aunque con «sobresaturación histórica» Nietzsche se refiere a la acumulación de conocimientos sobre el pasado de forma indiscriminada, en mi análisis estoy tratando del exceso de importancia que puede adquirir un suceso pasado concreto. La sobresaturación histórica nietzscheana se parecería más al caso de Funes el memorioso de Jorge Luis Borges que al de Chow. Sin embargo, aún así, creo que pueden servir de alguna manera para comprender los problemas que el abuso nostálgico de la memoria produce en 2046.

22 F. Nietzsche, op. cit., p. 76. 
convirtiéndoles en meros imitadores de lo que ya conocen ${ }^{23}$ y provocándose así la misma situación señalada por Benjamin y Todorov, sólo que atribuida a otras causas.

Mientras que para Benjamin y Todorov el problema radica en las mismas características que posee el método elegido para abordar el pasado, para Nietzsche se trata de un problema debido a los propios individuos, a su incapacidad para soportar la historia, para asimilarla, para convertir en sangre nueva «todo lo pasado, propio y extraño», ${ }^{24}$ tal y como haría un hombre con una fuerza plástica ${ }^{25}$ ilimitada. Y aquí nos encontramos con la capacidad de convertir el pasado en memoria de la que hablaba Braude. Un hombre con una fuerza plástica ilimitada no podría verse afectado por un abuso nostálgico de la memoria, ya que por muchas experiencias traumáticas que hubiera sufrido, las asimilaría obteniendo de ellas nuevos bríos para su vida presente. ${ }^{26}$ Pero los hombres no siempre poseen una fuerza plástica en un grado suficiente, y a veces, «a través de una única vivencia, de un único dolor, como resultado de una pequeña injusticia o de un minúsculo rasguño, se desangran incurablemente». ${ }^{27} \mathrm{Y}$ por eso añade que:

[...] sólo las personalidades fuertes pueden soportar la historia; los débiles son barridos completamente por ella. Esto se debe a que la historia confunde el sentimiento y la sensación donde éstos no son lo suficientemente fuertes para medirse con el pasado. Quien no se atreve ya más a confiar en sí mismo e involuntariamente pide consejo a la historia para comprender sus sentimientos - ¿icómo debo sentir aquí?»-, se convertirá progresivamente, por puro temor, en un actor que representa un papel..$^{28}$

En este sentido, la explicación del problema de Chow no se debería agotar atribuyéndola simplemente al hecho de abusar de la memoria nostálgica, sino

23 «Aquí y allá se llega incluso más lejos: al cinismo. Un cinismo que justifica la marcha entera de la Historia e incluso del desarrollo total del mundo para el propio uso del hombre moderno, es decir, como el canon cínico: todo tuvo exactamente que ocurrir como justo es ahora y de ningún modo podría haber sido el hombre diferente a como ya es; frente a este imperativo, nadie puede rebelarse». Ibid., p. 115 .

24 Ibid., p. 44.

25 «Esa fuerza para crecer por sí misma, ese poder de transformar y asimilar lo pasado y extraño, de sanar las heridas, de reemplazar lo perdido, de regenerar las formas destruidas...». Ibid., p. 43.

26 En otro lugar analicé la conexión que podría darse entre este uso de la fuerza plástica y un abuso analógico de la memoria, situación que traería sus propias consecuencias negativas. Para más información $c f$. A. Muñoz Corcuera 2010.

27 F. Nietzsche, op. cit., p. 43.

28 Ibid., p. 81. 
que los motivos de su desgracia habría que buscarlos en su debilidad como individuo, a su falta de fuerza plástica. Para Nietzsche sería esta falta de fuerza la causante de que Chow no pudiese utilizar la memoria nostálgica sin caer en su abuso, lo que le lleva a actuar de forma inmadura y a representar un papel, a sentirse una mera sombra o epígono de lo que él mismo fue en el pasado y a adoptar una actitud cínica frente a un presente que él considera algo inevitable pero que en realidad él mismo provoca. Y estas tres actitudes, como hemos visto anteriormente, desembocan en el hecho de que el presente y el futuro se convierten para Chow en una repetición del pasado.

La pregunta que cabe hacerse, no obstante, es por la existencia de esa fuerza plástica a la que Nietzsche se refiere. Y de corresponder esa idea efectivamente con una realidad, aún habría que preguntarse entonces por la posibilidad de la existencia de un hombre con la suficiente fuerza plástica como para asimilar cualquier pasado. Parece existir entre los individuos una cierta diferencia a la hora de asimilar los traumas del pasado para convertirlos en memoria-capacidad que podemos atribuir a la fuerza plástica nietzscheana-, así como también parece existir un límite en las situaciones traumáticas que son capaces de asimilar conservando la normalidad. Por eso el uso nostálgico del pasado siempre debe estar bajo sospecha, ya que la amenaza de caer en su abuso es permanente. ${ }^{29}$

Veamos cómo afecta todo esto a la noción de identidad. El filósofo catalán Manuel Cruz señala que la identidad no es una elección personal, sino una imposición. ${ }^{30}$ Pero una imposición que no viene sólo de fuera, de los otros, sino también de nuestro propio pasado. En este sentido, para Todorov en la identidad presente interviene por un lado un «yo arcaico», formado por nuestros recuerdos, y por otro un «yo reflexivo», imagen de la imagen que los demás tienen de nosotros. ${ }^{31}$ La identidad de Chow está marcada, principalmente, por sus recuerdos. La experiencia narrada en Deseando amar influyó profundamente en su vida, moviéndole a repetir compulsivamente el abandono sufrido en cada nueva relación que establece. De este modo, su identidad ha adquirido también una cierta resistencia al cambio, quedando en parte detenida, ya que sus nuevas relaciones son siempre repeticiones de esa experiencia anterior. Siendo así, su identidad no puede desarrollarse, pues todas las nuevas experiencias que podrían hacerle evolucionar no son más que repeticiones de sus experiencias pasadas, incapaces de producir nada nuevo. El excesivo peso de la memoria en la vida de Chow se constituye así en una amenaza para el desarrollo de su

29 «Se podrá decir entonces, en una primera aproximación, que la memoria literal, sobre todo si es llevada al extremo, es portadora de riesgos, mientras que la memoria ejemplar es potencialmente liberadora». T. Todorov, op. cit., p. 31.

30 M. Cruz 2005, pp. 83-84.

31 T. Todorov, op. cit., pp. 25-26. 
identidad en un sentido parecido - que no idéntico-al del caso de las personas que sufren amnesia anterógrada: ${ }^{32}$ mientras la identidad de estas personas se ve congelada al ser incapaces de recordar cualquier experiencia vivida con posterioridad al trauma sufrido, privándose así de la vida en el presente, la de Chow se ve detenida porque las nuevas experiencias que vive son repeticiones de la experiencia pasada. Este tipo de amnésicos viven en el pasado, por lo que conservan intacta la identidad del pasado, su personalidad no continúa desarrollándose. Chow vive en el presente, pero al ser éste una repetición del pasado, su identidad permanece inmutable.

De este modo el tejido del tiempo que Wong Kar-Wai nos muestra en 2046 se revela como la fuente de todos los problemas de Chow. Su vida inserta en el tiempo presente le es completamente ajena, pues su mirada se encuentra en un pasado que revive a cada instante con desesperación. Un pasado que-como en la cita inicial de Nietzsche- vuelve como un fantasma, sin ser visto, para envolver el presente y el futuro en un halo que condena a Chow no sólo a repetir constantemente la ruptura de su amor, sino también, y como consecuencia, a continuar siendo siempre el mismo hombre abandonado que aparecía al final de Deseando amar. El peligro del abuso de la memoria nostálgica nos hace pensar en la necesidad del olvido para la vida, no sólo para asegurar la continuidad de la identidad, sino también su desarrollo, pues la obsesión por el pasado sólo puede conducir a su repetición en el presente. Como señala Marc Augé:

El olvido nos devuelve al presente, aunque se conjugue en todos los tiempos: en futuro, para vivir el inicio; en presente, para vivir el instante; en pasado, para vivir el retorno; en todos los casos, para no repetirlo. Es necesario olvidar para estar presente, olvidar para no morir, olvidar para permanecer siempre fieles. ${ }^{33}$

32 Se trata de un tipo concreto de amnesia, generalmente producida por traumatismos craneales que implican lesiones cerebrales, en que se produce una incapacidad para almacenar nuevos recuerdos a partir del momento en que se produce el traumatismo. El ejemplo más famoso en el cine de este tipo de amnesia es el de Memento (C. Nolan, dir., 2000).

33 M. Augé 1998, p. 104. 


\section{REFERENCIAS BIBLIOGRÁFICAS}

AUGÉ, M. 1998: Las formas del olvido. Barcelona: Gedisa.

BENJAMIN, W. 2006: «Sobre el concepto de historia» en R. Mate, Medianoche en la historia: Comentarios a las tesis de Walter Benjamin «Sobre el concepto de historia». Madrid: Trotta.

BRAUDE, D. 2005: «2046: La mística poética de Wong Kar-wai». En: <http://www. imaginacionatrapada.com.ar/Cine/2046.htm>

BRAUDE, D. 2007: «Wong Kar-wai y su trilogía no habrá más penas ni olvidos». En: <http://www.imaginacionatrapada.com.ar/Cine/trilogia-wong-kar-wai.htm>

BORGES, J. L. 2002: «Funes el memorioso», en Ficciones. Madrid: Alianza.

CRUZ, M. 2005: Las malas pasadas del pasado: Identidad, responsabilidad, historia. Barcelona: Anagrama.

DENNETT, D. C. 1995: La conciencia explicada: Una teoría interdisciplinar. Barcelona: Paidós.

FREUD, S. 2006a: «Recuerdo, repetición y elaboración», en Obras Completas, vol. 3. Barcelona: RBA.

FREUD, S., 2006b: «Duelo y melancolía» en Obras Completas, vol. 3. Barcelona: RBA.

GERGEN, K. J. 1992: El yo saturado. Barcelona: Paidós.

GÓMEZ TARÍN, F. J. 2008: Wong Kar-wai: Grietas en el espacio-tiempo. Madrid: Akal.

KANDEL, E. R. 2007: En busca de la memoria: el nacimiento de una nueva ciencia de la mente. Buenos Aires: Katz.

KOSELLECK, R. 1993: Futuro pasado: para una semántica de los tiempos históricos. Barcelona: Paidós.

MATE, R. 2006: Medianoche en la historia: Comentarios a las tesis de Walter Benjamin «Sobre el concepto de historia». Madrid: Trotta.

MUÑOZ CORCUERA, A. 2010: «El caso de Memento: Una memoria nietzscheana en el cine postmoderno», Bajo Palabra. Revista de Filosofía, 4, pp. 181-190.

NIETZSCHE, F. 2003: Sobre la utilidad y el perjuicio de la historia para la vida. Madrid: Biblioteca Nueva.

PARFIT, D. 2004: Razones y personas. Boadilla del Monte: A. Machado Libros.

RICOEUR, P. 1999: La lectura del tiempo pasado: memoria y olvido. Madrid: Arrecife: Ediciones Universidad Autónoma, D. L.

SHOEMAKER, S. 1970: «Persons and Their Pasts», American Philosophical Quarterly, 7(4), pp. 269-285.

TODOROV, T. 2000: Los abusos de la memoria. Barcelona: Paidós.

\section{FILMOGRAFÍA}

KAR-WAI, W. (dir.) 1990: Días Salvajes (Days of Being Wild). China: In-Gear Film.

KAR-WAI, W. (dir.) 2000: Deseando amar (In the Mood for Love). China: Block 2 Pictures / Paradis Films / Jet Tone.

KAR-WAI, W. (dir.) 2004: 2046. China: Block 2 Pictures / Paradis Films / Orly Films. 
NOLAN, C. (dir.) 2000: Memento. Estados Unidos: Newmarket / Summit Entertainment

SCOTT, R. (dir.) 1982: Blade Runner. Estados Unidos: Warner Bros. Pictures.

Alfonso Muñoz Corcuera es investigador en el Departamento de Teoría del Conocimiento, Estética e Historia del Pensamiento de la Universidad Complutense de Madrid

Línea de investigación:

El problema de la identidad personal, relacionado con aspectos narrativos y cinematográficos.

Publicaciones recientes:

(2010) «El caso de Memento: Una memoria nietzscheana en el cine postmoderno», Bajo Palabra. Revista de Filosofía, 4, pp. 181-190.

(2010) «Memoria, ficción y realidad en Mulholland Drive y Carretera Perdida de David Lynch», Espéculo: Revista de Estudios Literarios, 44. En:

http://www.ucm.es/info/especulo/numero44/dvlynch.html

Dirección electrónica: alfonso.m.corcuera@gmail.com 